Case Report

\title{
S. lugdunensis Native-Joint Septic Arthritis: Case Report and Review of the Literature
}

\author{
C. Danielle Tan, ${ }^{1}$ Donna Moritz, ${ }^{2}$ and Alfredo J. Mena Lora ${ }^{2}$ \\ ${ }^{1}$ University of Illinois College of Medicine at Chicago, Chicago, IL, USA \\ ${ }^{2}$ University of Illinois at Chicago, Division of Infectious Diseases, Department of Medicine, Chicago, IL, USA \\ Correspondence should be addressed to Alfredo J. Mena Lora; amenalor@uic.edu
}

Received 3 July 2017; Accepted 29 November 2017; Published 28 December 2017

Academic Editor: Paola Di Carlo

Copyright (c) 2017 C. Danielle Tan et al. This is an open access article distributed under the Creative Commons Attribution License, which permits unrestricted use, distribution, and reproduction in any medium, provided the original work is properly cited.

\begin{abstract}
Staphylococcus lugdunensis is a skin commensal classified as a coagulase-negative Staphylococcus (CoNS). Though CoNS is typically associated with less aggressive clinical disease than Staphylococcus aureus, there is growing awareness that $S$. lugdunensis may be as virulent as $S$. aureus. The association between S. lugdunensis and infective endocarditis is well known, but few reports of native-joint disease with this organism exist. We report a case a 28 -year-old male with no prior medical problems presenting with native-joint septic arthritis. Cultures grew S. lugdunensis. To our knowledge, this is the fifth case reported in the literature.
\end{abstract}

\section{Introduction}

S. lugdunensis was first described in 1988 [1]. It remains a rare pathogen, but it has since been associated with a wide variety of clinical infectious syndromes, including cardiovascular, soft-tissue, bone, and prosthetic joint infections. S. lugdunensis has been associated with higher severity and mortality than other coagulase-negative Staphylococcus (CONS) species, presenting with features more similar to disease caused by $S$. aureus. A case series with native valve infective endocarditis (IE) caused by S. lugdunensis reported mortality as high as $42 \%$ and surgical needs for more than $51 \%$ of cases [2-5]. For prosthetic valve IE, mortality exceeded $78 \%$. Soft-tissue infections and pyogenic disease involving S. lugdunensis have also been reported and may be more common than once thought in community-acquired infections [6]. $S$. lugdunensis and $S$. aureus can infect joints via direct inoculation or hematogenous spread. The association between S. lugdunensis and prosthetic joint disease is well established, with reports of infections ranging between 6 weeks and 4 years after implantation $[4,7,8]$. There is a paucity of reports on infections involving native joints.

\section{Case Presentation}

A 28-year-old male with no past medical history presented to the emergency room with two weeks of right knee pain. He denied any recent sexually transmitted diseases, illicit drug use, or prior episodes of knee pain. He reports no trauma to the knee. Over-the-counter pain medications did not relieve his pain, which progressed and eventually limited his ability to use his joint.

On examination, the patient was afebrile. His right knee was erythematous, warm, swollen, and with reduced range of motion. A moderate effusion was noticed. He was unable to bare weight on the affected leg. Laboratory analysis revealed leukocytosis, with white blood cell counts of 12,100 per microliter ( $\mathrm{mcL}$ ). Imaging revealed mild softtissue swelling and a moderate effusion. A diagnostic arthrocentesis was performed, and fluid analysis revealed 28,875 white blood cells per $\mathrm{mcL}$, predominantly neutrophilic. Gram stain revealed Gram-positive cocci in clusters at 1 day of growth. At 48 hours, cultures grew S. lugdunensis. Magnetic resonance imaging (MRI) revealed diffuse soft-tissue edema, most prominent in the popliteal fossa around the knee and a large knee joint effusion with synovitis (Figure 1). Six synovial fluid 


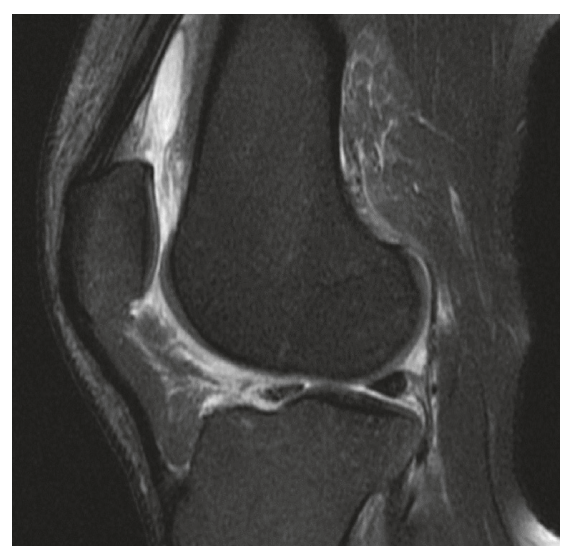

(a)

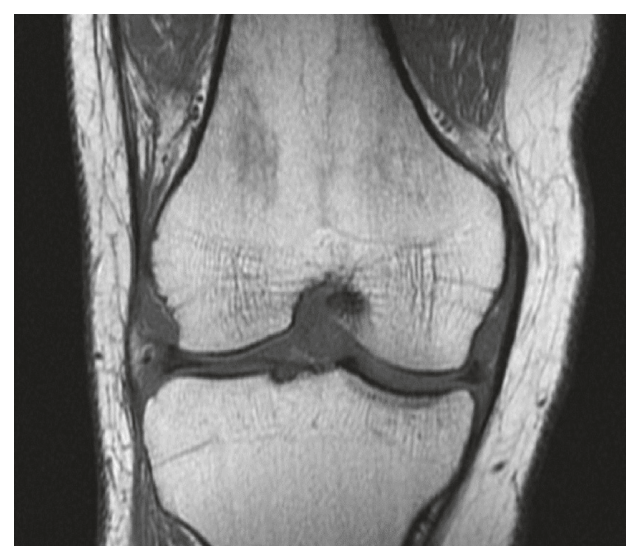

(b)

FIgURE 1: MRI of the knee in (a) T2 sagittal view and (b) T1 coronal view.

TABle 1: Management of S. lugdunensis.

\begin{tabular}{|c|c|c|c|c|}
\hline Case & $\begin{array}{c}\text { Trauma or joint } \\
\text { disease }\end{array}$ & $\begin{array}{l}\text { Antimicrobial } \\
\text { regimen }\end{array}$ & $\begin{array}{l}\text { Duration in } \\
\text { days }\end{array}$ & Surgical management \\
\hline Begly et al. [9] & None & $\begin{array}{l}\text { Vancomycin } \\
\text { Rifampin }\end{array}$ & 28 & $\begin{array}{l}\text { Open medial parapatellar arthrotomy with } \\
\text { irrigation and debridement on day } 1\end{array}$ \\
\hline Grupper et al. [7] & None & $\begin{array}{l}\text { Cefazolin } \\
\text { Rifampicin }\end{array}$ & 26 & $\begin{array}{l}\text { Daily joint aspiration from day } 1-3 \\
\text { Arthroscopy with debridement on day } 9 \text { and } 12\end{array}$ \\
\hline Kragsbjerg et al. [11] & Rheumatoid arthritis & Cloxacillin IV & 42 & Not specified \\
\hline Rose et al. [10] & Rheumatoid arthritis & Flucloxacillin IV & 112 & Joint irrigation and washout \\
\hline
\end{tabular}

samples grew S. lugdunensis. Susceptibilities were obtained via bioMerieux VITEK 2 antimicrobial susceptibility testing, showing resistance only to oxacillin.

The patient underwent washout by orthopedic surgery on day one and day three. Vancomycin was given for four days and subsequently changed to clindamycin. The patient completed seven days of parenteral antibiotics and transitioned to oral clindamycin for the remainder of a four-week course. He had full clinical resolution at his third week of therapy.

\section{Discussion and Literature Review}

Native-joint septic arthritis caused by S. lugdunensis is rare. Our review of the English literature found only four cases [7, 9-11]. One case did not have prior joint disease or chronic medical conditions, while all other cases had rheumatoid arthritis (RA) [7, 9-11]. Preexisting joint disease is a known risk factor for bacterial arthritis [12]. One prospective study of bacterial arthritis reported preexisting joint disease in $40 \%$ of cases, most commonly RA [13]. The most common pathogen associated with bacterial arthritis is $S$ aureus [12]. However, the incidence of CoNS has risen in the past two decades and is more common with prosthetic joints. An observational study with 7275 total hip and knee arthroplasties over 38 years found 75 cases of prosthetic joint infections, with CoNS causing 18 infections, 3 of which were S. lugdunensis [14]. The incidence of CoNS increased from 10 to $21 \%$ during period [15].
S. lugdunensis is classified as a CoNS. It nevertheless retains partial coagulase activity and has many similarities with $S$. aureus $[4,8]$. Agglutination tests are typically used to distinguish CoNS from $S$. aureus, but performance characteristics of these tests for S. lugdunensis are variable and can lead to false identification of $S$. aureus $[4,16]$. Thus, the true burden of $S$. lugdunensis may be underappreciated. Samples may be reported as $S$. aureus, and both pathogens cause similar disease. Both organisms are skin commensals and are associated with soft-tissue infections, bacteremia, and infective endocarditis. Like $S$ aureus, S. lugdunensis can cause severe disease. Infective endocarditis by S. lugdunensis can present with complications such as valvular perforation, myocardial abscesses, embolic stroke, and death $[2,4,11]$. Soft-tissue infections can be purulent and may progress to bacteremia and infective endocarditis $[10,11]$.

There are no randomized controlled data to guide the treatment of bacterial septic arthritis, and there is a paucity of data on S. lugdunensis infections. Three cases reported in the literature underwent joint irrigation, debridement, or other surgical interventions, and one did not specify (Table 1) [7, 9-11]. Antimicrobial courses included beta-lactams, glycopeptides, or lincomycins [7, 9-11]. Success with rifamycin combination therapy has also been reported [7, 9].

\section{Conclusion}

The present case contributes to a small but growing list of cases of native-joint septic arthritis by S. lugdunensis. 
Clinicians must be aware of the association between S. lugdunensis and severe clinical disease. Lincomycin-based therapy was a successful treatment option for our case. Further studies are needed to compare different therapeutic options.

\section{Conflicts of Interest}

The authors declare that there are no conflicts of interest regarding the publication of this paper.

\section{References}

[1] J. Freney, Y. Brun, M. Bes et al., "Staphylococcus lugdunensis sp. nov. and Staphylococcus schleiferi sp. nov., two species from human clinical specimens," International Journal of Systematic Bacteriology, vol. 38, no. 2, pp. 168-172, 1988.

[2] M. David, M. Loftsgaarden, and F. Chukwudelunzu, "Embolic stroke caused by Staphylococcus lugdunensis endocarditis complicating vasectomy in a 36-year-old man," Texas Heart Institute Journal, vol. 42, no. 6, pp. 585-587, 2015.

[3] G. De Hondt, M. Ieven, C. Vandermersch, and J. Colaert, "Destructive endocarditis caused by Staphylococcus lugdunensis. Case report and review of the literature," Acta Clinica Belgica, vol. 52, no. 1, pp. 27-30, 1997.

[4] A. Klotchko, M. R. Wallace, C. Licitra, and B. Sieger, "Staphylococcus lugdunensis: an emerging pathogen," Southern Medical Journal, vol. 104, no. 7, pp. 509-514, 2011.

[5] I. Anguera, A. Del Río, J. M. Miró et al., "Staphylococcus lugdunensis infective endocarditis: description of 10 cases and analysis of native valve, prosthetic valve, and pacemaker lead endocarditis clinical profiles," Heart, vol. 91, no. 2, p. e10, 2005.

[6] S. Böcher, B. Tønning, R. L. Skov, and J. Prag, "Staphylococcus lugdunensis, a common cause of skin and soft tissue infections in the community," Journal of Clinical Microbiology, vol. 47, no. 4, pp. 946-950, 2009.

[7] M. Grupper, I. Potasman, I. Rosner, G. Slobodin, and M. Rozenbaum, "Septic arthritis due to Staphylococcus lugdunensis in a native joint," Rheumatology International, vol. 30, no. 9, pp. 1231-1233, 2010.

[8] P. Sampathkumar, D. R. Osmon, and F. R. Cockerill, "Prosthetic joint infection due to Staphylococcus lugdunensis," Mayo Clinic Proceedings, vol. 75, no. 5, pp. 511-512, 2000.

[9] J. P. Begly, M. Sobieraj, F. A. Liporace, and A. Dayan, "Staphylococcus lugdunensis septic arthritis of a native knee: a case report," Bulletin of the Hospital for Joint Disease, vol. 74, no. 4, pp. 314-317, 2016.

[10] A. M. Rose, J. Barnett, S. Morris-Jones, and D. J. B. Marks, "Staphylococcus lugdunensis septic arthritis and epidural abscess in a patient with rheumatoid arthritis receiving antitumour necrosis factor therapy," Rheumatology, vol. 53, no. 12, p. 2231, 2014.

[11] P. Kragsbjerg, J. Bomfim-Loogna, E. Törnqvist, and B. Söderquist, "Development of antimicrobial resistance in Staphylococcus lugdunensis during treatment-report of a case of bacterial arthritis, vertebral osteomyelitis and infective endocarditis," Clinical Microbiology and Infection, vol. 6, no. 9, pp. 496-499, 2000.

[12] D. L. Goldenberg, "Septic arthritis and other infections of rheumatologic significance," Rheumatic Diseases Clinics of North America, vol. 17, no. 1, pp. 149-156, 1991.
[13] C. J. E. Kaandorp, P. Krijnen, H. J. B. Moens, J. D. F. Habbema, and D. van Schaardenburg, "The outcome of bacterial arthritis: a prospective community-based study," Arthritis \& Rheumatism, vol. 40, no. 5, pp. 884-892, 1997.

[14] G. Tsaras, D. R. Osmon, T. Mabry et al., "Incidence, secular trends, and outcomes of prosthetic joint infection: a populationbased study, Olmsted county, Minnesota, 1969-2007," Infection Control \& Hospital Epidemiology, vol. 33, no. 12, pp. 1207-1212, 2012.

[15] J. J. Dubost, M. Soubrier, C. De Champs et al., "No changes in the distribution of organisms responsible for septic arthritis over a 20 year period," Annals of the Rheumatic Diseases, vol. 61, no. 3, pp. 267-269, 2002.

[16] M. Mateo, J.-R. Maestre, L. Aguilar et al., "Genotypic versus phenotypic characterization, with respect to susceptibility and identification, of 17 clinical isolates of Staphylococcus lugdunensis," Journal of Antimicrobial Chemotherapy, vol. 56, no. 2, pp. 287-291, 2005. 


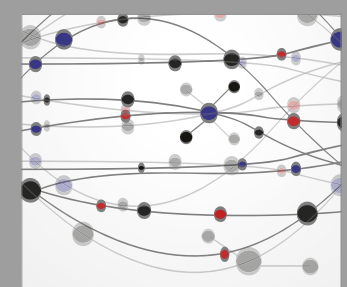

The Scientific World Journal
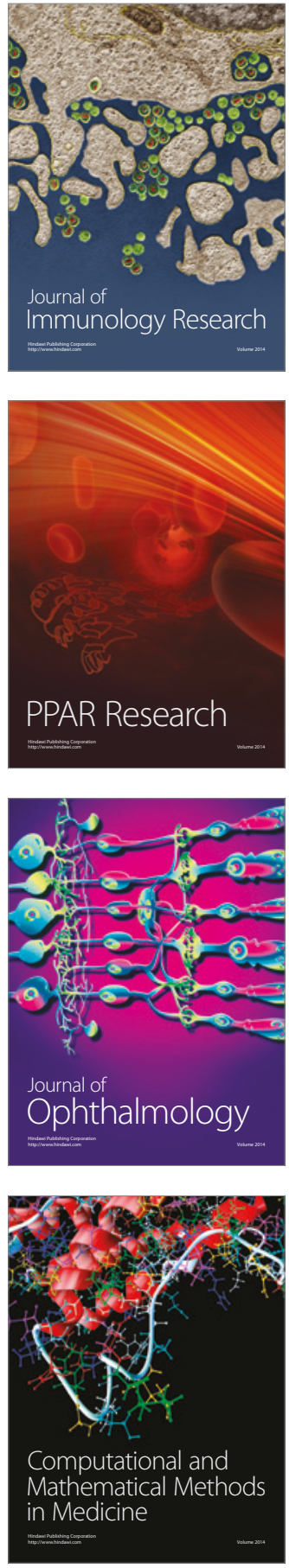

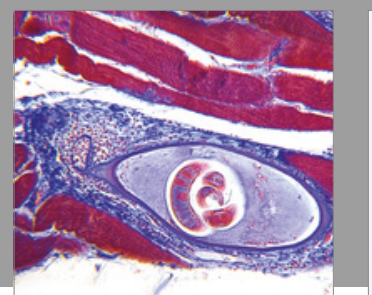

Gastroenterology Research and Practice
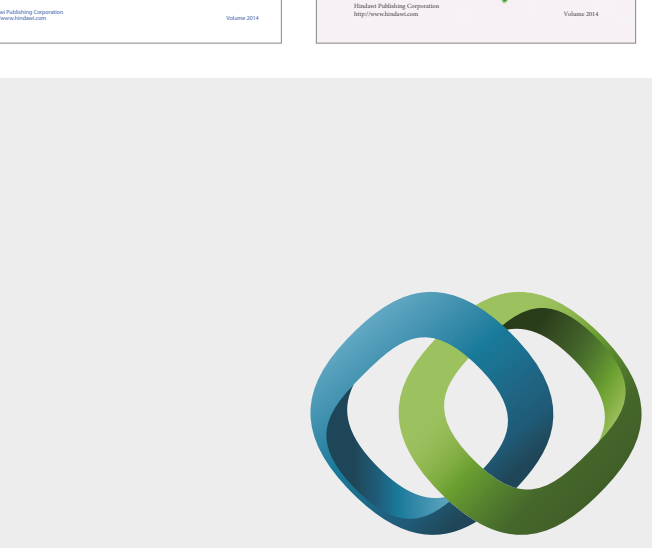

\section{Hindawi}

Submit your manuscripts at

https://www.hindawi.com
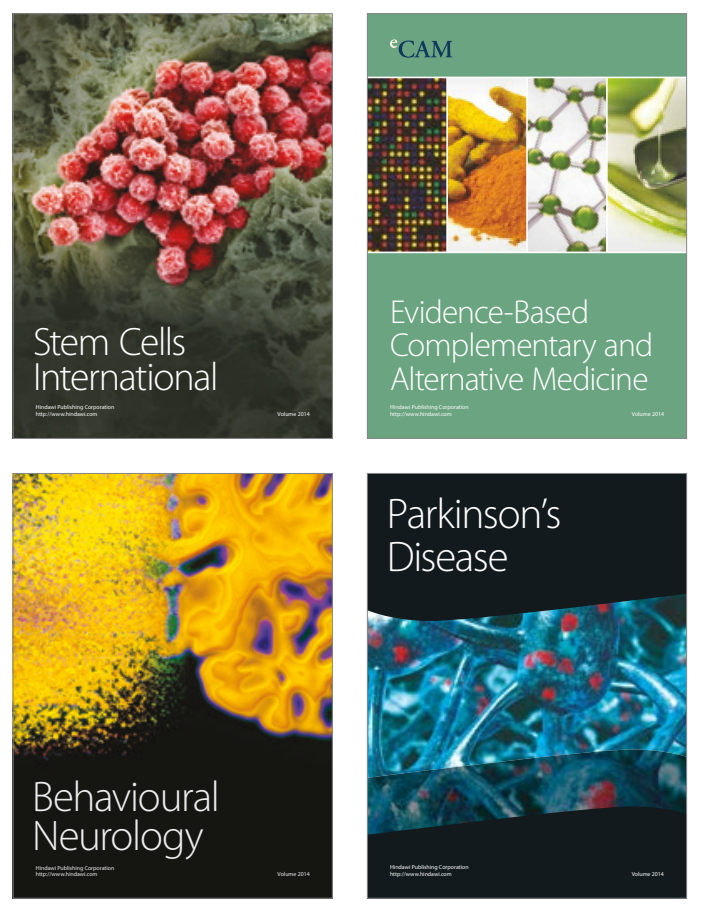
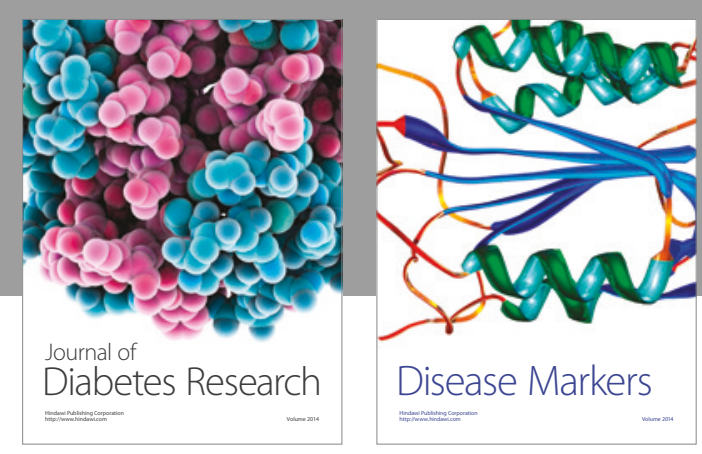

Disease Markers
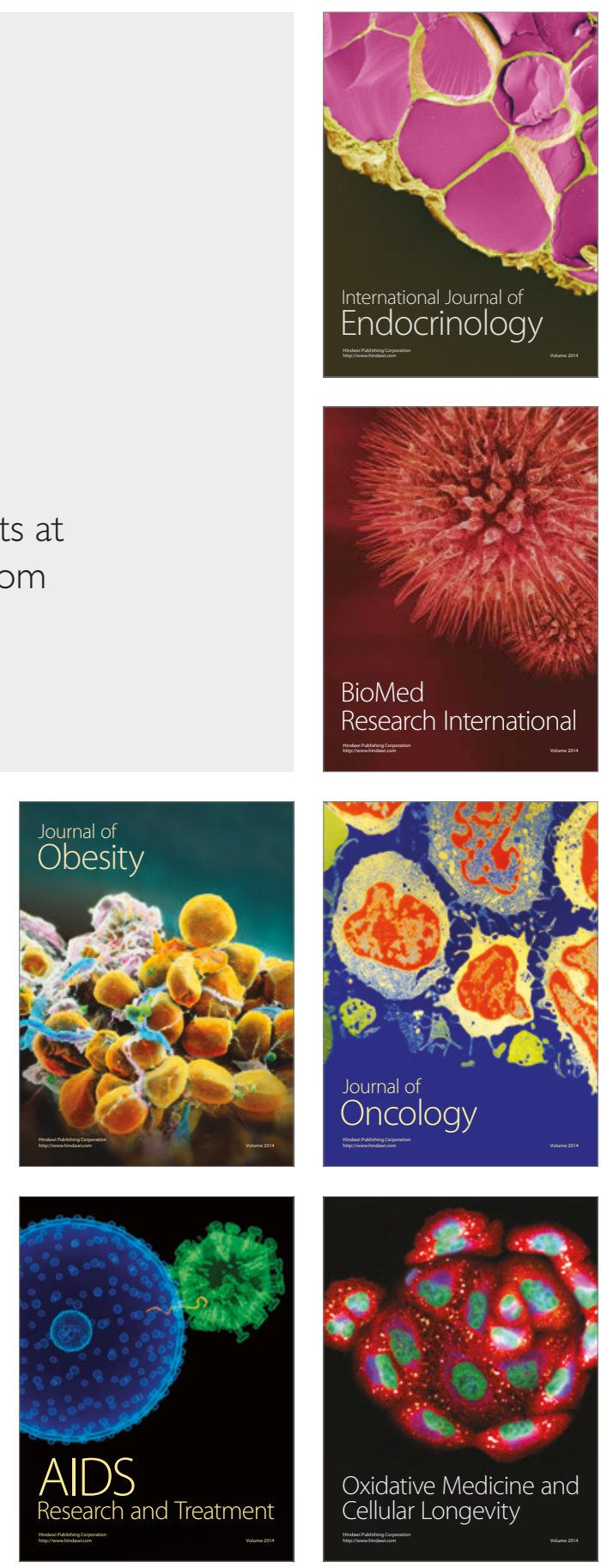